\title{
On the Smith Reduction Theorem for Almost Periodic ODEs Satisfying the Squeezing Property
}

\section{M. Anikushin}

We give a supplement to the Smith reduction theorem for nonautonomous ordinary differential equations (ODEs) that satisfy the squeezing property in the case when the right-hand side is almost periodic in time. The reduction theorem states that some set of nice solutions (including the bounded ones) of a given nonautonomous ODE satisfying the squeezing property with respect to some quadratic form can be mapped one-to-one onto the set of solutions of a certain system in the space of lower dimensions (the dimensions depend on the spectrum of the quadratic form). Thus, some properties of bounded solutions to the original equation can be studied through this projected equation. The main result of the present paper is that the projected system is almost periodic provided that the original differential equation is almost periodic and the inclusion for frequency modules of their right-hand sides holds (however, the right-hand sides must be of a special type). From such an improvement we derive an extension of Cartwright's result on the frequency spectrum of almost periodic solutions and obtain some theorems on the existence of almost periodic solutions based on low-dimensional analogs in dimensions 2 and 3. The latter results require an additional hypothesis about the positive uniformly Lyapunov stability and, since we are interested in nonlinear phenomena, our existence theorems cannot be directly applied. On the other hand, our results may be applicable to study the question of sensitive dependence on initial conditions in an almost periodic system with a strange nonchaotic attractor. We discuss how to apply this kind of results to the Chua system with an almost periodic perturbation. In such a system the appearance of regular almost periodic oscillations as well as strange nonchaotic and chaotic attractors is possible.

Keywords: almost periodic function, dimension theory, squeezing property, strange nonchaotic attractor

Received January 21, 2019

Accepted March 21, 2019

This work is supported by the Leading Scientific Schools of Russia (project NSh-2858.2018.1).

Mikhail M. Anikushin

demolishka@gmail.com

Saint-Petersburg State University

Universitetskiy pr. 28, Peterhof, Saint-Petersburg, 198504 Russia 


\section{Introduction}

\subsection{Background}

Consider the following ordinary differential equation:

$$
\dot{u}=f(t, u) \text {, }
$$

where $f: \mathbb{R} \times \mathbb{R}^{n} \rightarrow \mathbb{R}^{n}$ is continuous. We denote the Euclidean scalar product in $\mathbb{R}^{n}$ by $(\cdot, \cdot)$ and the corresponding Euclidean norm of a vector or matrix by $|\cdot|$. The following conditions for (1.1) will be considered.

(A1) For every compact set $\mathcal{K} \subset \mathbb{R}^{n}$ there is a constant $C=C(\mathcal{K})>0$ such that $|f(t, u)-f(t, v)| \leqslant C|u-v|$ for all $u, v \in \mathcal{K}$ and $t \in \mathbb{R}$.

(A2) Each maximal solution $u(t)=u\left(t, t_{0}, u_{0}\right)$ of (1.1) with initial conditions $\left(t_{0}, u_{0}\right)$ exists at least on $\left[t_{0},+\infty\right)$.

(A3) There exist constants $\delta>0$ and $\nu>0$ and a constant real symmetric $n \times n$-matrix $P$ such that for all $t \in \mathbb{R}$ and $u, v \in \mathbb{R}^{n}$ we have

$$
(P(u-v), f(t, u)-f(t, v)+\nu(u-v)) \leqslant-\delta|u-v|^{2} .
$$

(A4) The matrix $P$ has $j>0$ negative and $n-j$ positive eigenvalues.

In [13] it was shown (see Corollary 8.1 therein) that under conditions (A1)-(A4) and (A6) (see below) there exist a continuous function $\Phi^{(0)}: \mathbb{R} \times \mathbb{R}^{j} \rightarrow \mathbb{R}^{n}$ and a linear map $\Pi: \mathbb{R}^{n} \rightarrow \mathbb{R}^{j}$ such that "nice" solutions (called amenable, see Section 2) to (1.1) are mapped one-to-one by $\Pi$ onto the solutions of the following equation ${ }^{1}$ in $\mathbb{R}^{j}$ :

$$
\dot{\zeta}=\Pi f\left(t, \Phi^{(0)}(t, \zeta)\right),
$$

and if $f$ is $T$-periodic in time, then so is $\Phi^{(0)}$ and, consequently, the right-hand side of (1.3). The latter led to an extension of results of Massera (on the existence of periodic solutions ${ }^{2}$ ) and Cartwright (on the size of the Fourier spectrum of almost periodic solutions). In the present paper we study the almost periodicity of the right-hand side in (1.3) in the case when the original differential equation (1.1) is almost periodic and show some consequences of our investigation. In Section 3 we discuss some applications of our results to the question of sensitive dependence on initial conditions in a system with a strange nonchaotic attractor. In particular, an almost periodically forced Chua system will be studied. In [15] it was shown that for certain parameters the appearance of strange nonchaotic attractors in such a system is possible.

Conditions (A3) (the squeezing property ${ }^{3}$ ) and (A4) can be effectively verified for a certain class of control systems by using frequency-domain methods (see [13] and [3]). For various applications of frequency-domain methods in the dimension theory of dynamical systems (including, in particular, the study of dimensional-like properties of almost periodic oscillations) we refer to $[1,2,8]$. The Smith method was developed for evolution equations in [11] using the Yakubovich-Likhtarnikov frequency theorem.

To make precise statements of our results, we need to introduce some concepts.

\footnotetext{
${ }^{1}$ For a precise definitions of $\Pi$ and $\Phi^{(0)}$, see Section 2.

${ }^{2}$ Later, these ideas were applied by Smith in [14] to study a class of periodic control systems with delay.

${ }^{3}$ The term squeezing property comes from inertial manifold theory [12].
} 


\subsection{Fourier spectrum of almost periodic functions}

Recall that a subset $A \subset \mathbb{R}$ is called relatively dense if there is $L>0$ such that the set $[a, a+L] \cap A$ is not empty for any $a \in \mathbb{R}$.

Suppose $\mathbb{E}$ is a Banach space with the norm $\|\cdot\|$ and $u: \mathbb{R} \rightarrow \mathbb{E}$ is continuous. For a given $\varepsilon>0$ the number $\tau \in \mathbb{R}$ is called an $\varepsilon$-almost period of $u$ if $\sup _{t \in \mathbb{R}}\|u(t+\tau)-u(t)\| \leqslant \varepsilon$ holds. Denote the set of all $\varepsilon$-almost periods by $\mathcal{T}_{\varepsilon}(u)$. The function $u$ is called $\mathbb{E}$-almost periodic (or simply, almost periodic) if the set $\mathcal{T}_{\varepsilon}(u)$ is relatively dense for every $\varepsilon>0$.

For every $\mathbb{E}$-almost periodic function $u$ there is a formal Fourier series (see [9])

$$
u(t) \sim \sum_{k=1}^{\infty} U_{k} e^{i \lambda_{k} t},
$$

with the Fourier coefficients $U_{k} \in \mathbb{E}^{\mathbb{C}}$ and the Fourier exponents $\lambda_{k} \in \mathbb{R}$. Let $\bmod _{\mathbb{Z}}(u)$ be the least additive subgroup of $\mathbb{R}$ containing the Fourier exponents $\lambda_{1}, \lambda_{2}, \ldots$. The $\operatorname{group}_{\bmod _{\mathbb{Z}}}(u)$ is the $\mathbb{Z}$-module of $u$. The least $\mathbb{Q}$-vector space in $\mathbb{R}$ containing $\lambda_{1}, \lambda_{2}, \ldots$ is called the $\mathbb{Q}$ module of $u(\cdot)$ and is denoted by $\bmod _{\mathbb{Q}}(u)$. For two almost periodic functions, say $u$ and $v$, it is convenient to use a subgroup $\bmod _{\mathbb{Z}}(u, v)$ generated by the union of their Fourier exponents. The set $\bmod _{\mathbb{Q}}(u, v)$ can be introduced analogously.

Now consider the following assumptions.

(A5) The function $f(\cdot, u)$ is almost periodic uniformly in $u$ from compact subsets of $\mathbb{R}^{n}$.

(A6) There exists at least one amenable solution to (1.1).

Remark 1. Condition (A5) means that $f$ is almost periodic as a function $t \mapsto f(t, \cdot) \in C\left(\mathcal{K} ; \mathbb{R}^{n}\right)$ for every compact $\mathcal{K} \subset \mathbb{R}^{n}$. We say that the corresponding $\varepsilon$-almost periods are the $\varepsilon$-almost periods of $f(\cdot, u)$ uniformly in $u \in \mathcal{K}$. Since $C\left(\mathcal{K} ; \mathbb{R}^{n}\right)$ is a Banach space, we have the introduced Fourier theory for such functions, namely, one can write

$$
f(t, u) \sim \sum_{k=1}^{\infty} F_{k}(u) e^{i \lambda_{k}^{*} t}
$$

where $F_{k}(\cdot), k=1,2, \ldots$, are continuous functions that are not identically zero on $\mathbb{R}^{n}$. Thus, we can consider the $\mathbb{Z}$-module $\bmod _{\mathbb{Z}}(f)$ of $f$ generated by all the exponents from (1.5). In [4] Theorem 1 states that if $u(\cdot)$ is an almost periodic solution to (1.1) and $f(t, u(s))$ is independent of $t$ for every $s$, then the inclusion $\bmod _{\mathbb{Z}}(u) \supset \bmod _{\mathbb{Z}}(f)$ holds. We have to note that this cannot be true since some of the Fourier coefficients $F_{k}(\cdot)$ may vanish on the closure of $u(\cdot)$, i.e., on the set $\mathcal{M}_{u}=\mathrm{Clu}(\mathbb{R})$. From this observation a simple counterexample to the statement can be constructed. However, even if we consider the Fourier expansion of $f$ with the coefficients that do not vanish on $\mathcal{M}_{u}$. Theorem 1 from [4] in the claimed generality still seems to fail ${ }^{4}$. So, we have to emphasize what do we mean by such modules containments for functions like $f(t, u)$ to avoid any misunderstandings in the future. Namely, for a compact set $\mathcal{K}$ we denote by $\left.f\right|_{\mathcal{K}}$ the almost periodic function $t \mapsto f(t, \cdot) \in C\left(\mathcal{K} ; \mathbb{R}^{n}\right)$. It is clear that the $\operatorname{module}_{\bmod }\left(\left.f\right|_{\mathcal{K}}\right)$ is generated by the Fourier exponents from (1.5) for which the corresponding Fourier coefficients do not vanish on $\mathcal{K}$. Now suppose we have a function $g: \mathbb{R} \times \mathbb{R}^{j} \rightarrow \mathbb{R}^{j}$ and $g(\cdot, \zeta)$ is almost periodic in $t$ uniformly on $\zeta$ from compact subsets of $\mathbb{R}^{j}$. We use the notation $\bmod _{\mathbb{Z}}(g) \Subset \bmod _{\mathbb{Z}}(f)$ meaning that for every compact subset $\mathcal{K}_{1}$ in $\mathbb{R}^{j}$ there exists a compact subset $\mathcal{K}_{2}$ in $\mathbb{R}^{n}$ such that $\bmod _{\mathbb{Z}}\left(\left.g\right|_{\mathcal{K}_{1}}\right) \subset \bmod _{\mathbb{Z}}\left(\left.f\right|_{\mathcal{K}_{2}}\right)$.

\footnotetext{
${ }^{4}$ There is a one more scenario of "loss of exponents" (i.e., the conditions under which the $\mathbb{Z}$-module of the solution may not contain a given exponent of $f$ ) that may happen under the conditions of Theorem 1 in [4].
} 
Note that the property $\bmod _{\mathbb{Z}}(g) \Subset \bmod _{\mathbb{Z}}(f)$ is stronger than the inclusion $\bmod _{\mathbb{Z}}(g) \subset \bmod _{\mathbb{Z}}(f)$. Similar definitions may be given for the $\mathbb{Q}$-modules of such functions.

Unfortunately, we have to restrict the right-hand sides of (1.1) as in the following assumption (see also Remark 2 below).

(A5*) Suppose that $f(t, u)=h(u)+\stackrel{\circ}{f}(t)$, where $\stackrel{\circ}{f}(t)$ is $\mathbb{R}^{n}$-almost periodic.

\subsection{Main results}

The main theorem of this paper is as follows.

Theorem 1. Suppose that assumptions (A1)-(A4), (A5*) and (A6) hold; then the function $\Phi^{(0)}(\cdot, \zeta)$ is almost periodic uniformly in $\zeta$ from compact subsets of $\mathbb{R}^{j}$ and we have

$$
\bmod _{\mathbb{Z}}\left(\Phi^{(0)}\right) \Subset \bmod _{\mathbb{Z}}(\stackrel{\circ}{f}) .
$$

REMARK 2. It seems that for more general (than it is required in $\left(\mathbf{A} 5^{*}\right)$ ) right-hand sides the inclusion in (1.6) may fail even if we know that the function $\Phi^{(0)}$ is almost periodic. This is due to the fact that the function $\Phi^{(0)}$ operates with solutions and the almost periodic ones may have additional frequencies some of which $\Phi^{(0)}$ may inherit. However, the latter is impossible under condition $\left(\mathbf{A} 5^{*}\right)$. The specificity of that is in the last inequality in (2.9).

The proof of Theorem 1 is outlined in the next section. Now we are going to discuss some of its corollaries.

An almost periodic solution $u^{*}(\cdot)$ to (1.1) may have the Fourier exponents that do not belong to the $\mathbb{Q}$-module of $f$. It turns out that the dimension of the subspace generated by these additional exponents is bounded from above by $n-1$. Namely,

$$
\operatorname{dim} \frac{\bmod _{\mathbb{Q}}\left(u^{*}, f\right)}{\bmod _{\mathbb{Q}}(f)} \leqslant n-1 .
$$

The bound in (1.7) was shown by Cartwright (see [4]) and reproved by O'Brien (see [10]). As a corollary of our Theorem 1 we may sharp (1.7) as in the following theorem ${ }^{5}$.

Theorem 2. Assume that (A1)-(A4) and (A5*) are satisfied. Let $u^{*}(\cdot)$ be an almost periodic solution to $(1.1)$; then

$$
\operatorname{dim} \frac{\bmod _{\mathbb{Q}}\left(u^{*}, f\right)}{\bmod _{\mathbb{Q}}(f)} \leqslant j-1 .
$$

Moreover, the function $g(t, \zeta):=\Pi f\left(t, \Phi^{(0)}(t, \zeta)\right)$ is almost periodic and $\bmod _{\mathbb{Z}}(g) \subset \bmod _{\mathbb{Z}}(\stackrel{\circ}{f})$.

For the proof of Theorem 2 see the next section.

Analogously to Theorem 7 in [13], extending the results of Massera to periodic ODEs satisfying (A4) with $j=2$, due to our Theorem 1 we can obtain below the theorems on the existence of almost periodic solutions to (1.1). Here we use well-known results in low dimensions, namely, in dimensions 2 and 3 (see [9]). These theorems are stated and proved in the next section (see Theorems 3 and 4).

\footnotetext{
${ }^{5}$ The estimate in (1.8) can be shown by using only conditions (A2)-(A3) and without referring to the Smith projection theorem. To get this one has to follow Cartwright's proof in [4] to construct an almost periodic flow on the set of initial values of almost periodic solutions. The map $\Pi$ defined in the next section takes the flow into $\mathbb{R}^{j}$ (since the initial values belong to an amenable set on which $\Pi$ is a homeomorphism), where the Cartwright theorem can be used. In the proof of our Theorem 2 we establish some additional properties required for further investigations.
} 


\section{The proofs}

We need the following lemma from [7].

Lemma 1. Let $u: \mathbb{R} \rightarrow \mathbb{E}$ and $v: \mathbb{R} \rightarrow \mathbb{F}$ be two almost periodic functions with the values in some Banach spaces $\mathbb{E}$ and $\mathbb{F}$. The following conditions are equivalent:

1) $\bmod _{\mathbb{Z}}(u) \subset \bmod _{\mathbb{Z}}(v)$;

2) For every $\varepsilon>0$ there exists $\delta>0$ such that $\mathcal{T}_{\delta}(v) \subset \mathcal{T}_{\varepsilon}(u)$.

Along with (1.1) consider the family of ordinary differential equations for $\tau \in \mathbb{R}$

$$
\dot{u}=\sigma^{\tau} f(t, u)=f(t+\tau, u) .
$$

It is clear that conditions (A1)-(A4) are satisfied for $(2 . \tau)$. The solution $u(\cdot)$ to $(2 . \tau)$ is called amenable if it exists on the left semiaxis and

$$
\int_{-\infty}^{0} e^{2 \nu s}|u(s)|^{2} d s<\infty .
$$

Suppose $\mathfrak{A}_{t}\left(\sigma^{\tau} f\right):=\{u(t) \mid u(\cdot)$ is an amenable solution to $(2 . \tau)\}$. The set $\mathfrak{A}_{t}\left(\sigma^{\tau} f\right)$ is an amenable set for $(2 . \tau)$.

Put $V(u):=(P u, u)$, where $P$ is from (A3). By (A4) there exists a linear transformation $Q$ sending $(\zeta, \eta) \in \mathbb{R}^{j} \times \mathbb{R}^{n-j}$ to $u$ such that for $u=Q(\zeta, \eta)^{T}$ we have $V(u)=|\eta|^{2}-|\zeta|^{2}$. Define a linear map $\Pi: \mathbb{R}^{n} \rightarrow \mathbb{R}^{j}$ as $\Pi u:=\zeta$. Since $\left|Q^{-1} u\right|=|\zeta|^{2}+|\eta|^{2}$ we have

$$
V(u)+2|\Pi u|^{2}=\left|Q^{-1} u\right|^{2} \geqslant|\Pi u|^{2} .
$$

An important property of two amenable solutions $u(\cdot)$ and $v(\cdot)$ to $(2 . \tau)$ is that $V(u(t)-v(t)) \leqslant 0$ (see Lemma 1 on p. 687 in [13]). Putting $u=u(t) \in \mathfrak{A}_{t}\left(\sigma^{\tau} f\right), v=v(t) \in \mathfrak{A}_{t}\left(\sigma^{\tau} f\right), \Pi u(t)=\zeta_{1}$ and $\Pi v(t)=\zeta_{2}$ from $(2.2)$, we obtain

$$
\left|Q^{-1}\right|^{-2} \cdot\left|\zeta_{1}-\zeta_{2}\right|^{2} \leqslant|u-v|^{2} \leqslant 2|Q|^{2} \cdot\left|\zeta_{1}-\zeta_{2}\right|^{2} .
$$

From (2.3) it follows that $\Pi: \mathfrak{A}_{t}\left(\sigma^{\tau} f\right) \rightarrow \Pi \mathfrak{A}_{t}\left(\sigma^{\tau} f\right)$ is a homeomorphism. Theorem 8 in [13] states that under assumptions (A1)-(A4) and (A6) we have $\Pi \mathfrak{A}_{t}\left(\sigma^{\tau} f\right)=\mathbb{R}^{j}$ for all $t \in \mathbb{R}$ and $\tau \in \mathbb{R}$.

Now the map $\Phi^{(\tau)}(t, \zeta)$ is defined by the identity $\Phi^{(\tau)}(t, \Pi u)=u$ for every $u \in \mathfrak{A}_{t}(\tau)$. By Corollary 8.1 in [13] (which we call the Smith reduction theorem) the relations $\zeta(t)=\Pi u(t)$ and $u(t)=\Phi^{(\tau)}(t, \zeta(t))$ provide a one-to-one correspondence between the amenable solutions $u(\cdot)$ to $(2 . \tau)$ and the solutions $\zeta(\cdot)$ to the following equation in $\mathbb{R}^{j}$ :

$$
\dot{\zeta}(t)=\Pi \sigma^{\tau} f\left(t, \Phi^{(\tau)}(t, \zeta)\right)
$$

Now we are going to prove Theorem 1. 
Proof. It is clear that $u(\cdot)$ is an amenable solution to (1.1) iff $v(t):=u(t+\tau)$ is an amenable solution to $(2 . \tau)$. From this we get

$$
\mathfrak{A}_{t+\tau}(f)=\mathfrak{A}_{t}\left(\sigma^{\tau} f\right)
$$

One may rewrite $(2.5)$ in terms of $\Phi^{(\tau)}$ as

$$
\Phi^{(\tau)}(t, \zeta)=\Phi^{(0)}(t+\tau, \zeta), \text { for all } \zeta \in \mathbb{R}^{j} .
$$

Now fix $t_{0} \in \mathbb{R}$ and $\zeta_{0} \in \mathcal{K}_{1} \subset \mathbb{R}^{n}$ for a given compact set $\mathcal{K}_{1}$. From (2.6) we have

$$
\Phi^{(0)}\left(t_{0}+\tau, \zeta_{0}\right)-\Phi^{(0)}\left(t_{0}, \zeta_{0}\right)=\Phi^{(\tau)}\left(t_{0}, \zeta_{0}\right)-\Phi^{(0)}\left(t_{0}, \zeta_{0}\right) .
$$

So, there are amenable solutions $u(\cdot)$ of $(1.1)$ and $v(\cdot)$ of $(2 . \tau)$ such that $\Pi u\left(t_{0}\right)=\zeta_{0}, \Pi v\left(t_{0}\right)=\zeta_{0}$, $\Phi^{(0)}\left(t, \zeta_{0}\right)=u\left(t_{0}\right)$ and $\Phi^{(\tau)}\left(t_{0}, \zeta\right)=v\left(t_{0}\right)$. From (2.7) and (2.2) we have

$$
\begin{aligned}
\left|\Phi^{(0)}\left(t_{0}+\tau, \zeta_{0}\right)-\Phi^{(0)}\left(t_{0}, \zeta_{0}\right)\right|^{2} & =\left|u\left(t_{0}\right)-v\left(t_{0}\right)\right|^{2} \leqslant|Q|^{2}\left|Q^{-1}\left(u\left(t_{0}\right)-v\left(t_{0}\right)\right)\right|^{2} \\
& =|Q|^{2} \cdot\left[V\left(u\left(t_{0}\right)-v\left(t_{0}\right)\right)+2\left|\Pi\left(u\left(t_{0}\right)-v\left(t_{0}\right)\right)\right|^{2}\right] \\
& =|Q|^{2} \cdot V\left(u\left(t_{0}\right)-v\left(t_{0}\right)\right) .
\end{aligned}
$$

Suppose $\tau$ is an $\varepsilon$-almost period of $f$ from (A5*). From (A3) for any $s \in \mathbb{R}$ we deduce

$$
\begin{aligned}
\frac{d}{d s}\left(e^{2 \nu s} V(u(s)-v(s))\right) & =2 \nu e^{2 \nu s} V(u(s)-v(s))+2 e^{2 \nu s}(P(u(s)-v(s)), \dot{u}(s)-\dot{v}(s)) \\
& =2 e^{2 \nu s}(P(u(s)-v(s)), f(s, u(s))-f(s+\tau, v(s))+\nu(u(s)-v(s))) \\
& \leqslant-2 \delta e^{2 \nu s}|u(s)-v(s)|^{2}+2|P| \varepsilon e^{2 \nu s}|u(s)-v(s)| .
\end{aligned}
$$

Since the integral $\int_{-\infty}^{0} e^{2 \nu s}|u(s)-v(s)|^{2} d s$ converges there exists a sequence $t_{k} \rightarrow-\infty$ as $k \rightarrow \infty$ such that $e^{2 \nu t_{k}} V\left(u\left(t_{k}\right)-v\left(t_{k}\right)\right) \rightarrow 0$. Integrating $(2.9)$ on $\left[t_{k}, t_{0}\right]$ and taking it to the limit as $k \rightarrow \infty$ we get

$$
e^{2 \nu t_{0}} V\left(u\left(t_{0}\right)-v\left(t_{0}\right)\right) \leqslant-2 \delta \int_{-\infty}^{t_{0}} e^{2 \nu s}|u(s)-v(s)|^{2} d s+2|P| \varepsilon \int_{-\infty}^{t_{0}} e^{2 \nu s}|u(s)-v(s)| d s .
$$

Since the quadratic function $-a x^{2}+b x$, where $a, b>0$, reaches its maximum at $x=\frac{b}{2 a}$, we have

$$
V\left(u\left(t_{0}\right)-v\left(t_{0}\right)\right) \leqslant \frac{|P|^{2}}{\delta \nu} \varepsilon^{2} .
$$

From (2.8) and (2.11) we obtain

$$
\left|\Phi^{(0)}\left(t_{0}+\tau, \zeta_{0}\right)-\Phi^{(0)}\left(t_{0}, \zeta_{0}\right)\right|^{2} \leqslant|Q|^{2} V\left(u\left(t_{0}\right)-v\left(t_{0}\right)\right) \leqslant|Q|^{2} \cdot \frac{|P|^{2}}{\delta \nu} \cdot \varepsilon^{2} .
$$

Note that the last estimate is independent of $t_{0} \in \mathbb{R}$ and $\zeta_{0} \in \mathcal{K}_{1}$ (due to (A5*) it holds even for all $\left.\zeta_{0} \in \mathbb{R}^{j}\right)$. Therefore, $\tau$ is an $C \varepsilon$-almost period of $\Phi^{(0)}(\cdot, \zeta)$ for $C=|Q| \cdot|P| \cdot(\delta \nu)^{-1 / 2}$ uniformly in $\zeta \in \mathcal{K}_{1}$. From Lemma 1 it follows that $\bmod _{\mathbb{Z}}\left(\left.\Phi^{(0)}\right|_{\mathcal{K}_{1}}\right) \subset \bmod _{\mathbb{Z}}(f)$. Thus, the proof is finished.

The proof of Theorem 2 is as follows. 
Proof. Since $u^{*}(\cdot)$ is an almost periodic solution to (1.1) for $\zeta^{*}(t)=\Pi u^{*}(t)$, we have that $\zeta^{*}(\cdot)$ is almost periodic and the inclusion $\bmod _{\mathbb{Z}}\left(\zeta^{*}\right) \subset \bmod _{\mathbb{Z}}\left(u^{*}\right)$ holds due to Lemma 1.

Now let $\tau$ be an $\varepsilon$-almost period for $\zeta^{*}(\cdot)$ and $f$. Put $v^{*}(t):=u^{*}(t+\tau)$ and from (2.2) we have

$$
\left|u^{*}(t)-v^{*}(t)\right|^{2} \leqslant|Q|^{2}\left[2 \cdot|\zeta(t)-\zeta(t+\tau)|^{2}+V\left(u^{*}(t)-v^{*}(t)\right)\right] .
$$

As in the proof of Theorem 1 we can estimate $V\left(u^{*}(t)-v^{*}(t)\right) \leqslant C \varepsilon$ for some $C>0$. Therefore, Lemma 1 guarantees that $\bmod _{\mathbb{Z}}\left(u^{*}\right) \subset \bmod _{\mathbb{Z}}\left(\zeta^{*}, \stackrel{\circ}{f}\right)$ and, consequently, $\bmod _{\mathbb{Z}}\left(u^{*}, \stackrel{\circ}{f}\right)=$ $=\bmod _{\mathbb{Z}}\left(\zeta^{*}, \stackrel{\circ}{f}\right)$. Put $g(t, \zeta):=\Pi f\left(t, \Phi^{(0)}(t, \zeta)\right)$. Using $(2.2)$ we get

$$
\begin{aligned}
|g(t+\tau, \zeta)-g(t, \zeta)| & \leqslant\left|Q^{-1}\right| \cdot\left|f\left(t+\tau, \Phi^{(0)}(t+\tau, \zeta)\right)-f\left(t+\tau, \Phi^{(0)}(t, \zeta)\right)\right| \\
& +\left|Q^{-1}\right| \cdot\left|f\left(t+\tau, \Phi^{(0)}(t, \zeta)\right)-f\left(t, \Phi^{(0)}(t, \zeta)\right)\right|,
\end{aligned}
$$

and in virtue of $(\mathbf{A} 1)$ and $\left(\mathbf{A} 5^{*}\right)$ it follows that $\bmod _{\mathbb{Z}}(g) \Subset \bmod _{\mathbb{Z}}\left(\stackrel{\circ}{f}, \Phi^{(0)}\right)$ and, by Theorem 1 , $\bmod _{\mathbb{Z}}(g) \Subset \bmod _{\mathbb{Z}}(f)$. Therefore,

$$
\operatorname{dim} \frac{\bmod _{\mathbb{Q}}(u, f)}{\bmod _{\mathbb{Q}}(f)}=\operatorname{dim} \frac{\bmod _{\mathbb{Q}}(\zeta, f)}{\bmod _{\mathbb{Q}}(f)} \leqslant \operatorname{dim} \frac{\bmod _{\mathbb{Q}}(\zeta, g)}{\bmod _{\mathbb{Q}}(g)} \leqslant j-1,
$$

where the last inequality is due to Theorem 2 in [4].

To study the existence of almost periodic solutions we need to consider the limiting equations (Eqs. (2. $\hat{f})$ below). The hull $\mathcal{H}(f)$ of $f$ is the set of all functions $\hat{f}(t, u)$ such that for some sequence $\left\{t_{k}\right\}_{k=1}^{\infty}$ we have $f\left(t+t_{k}, u\right) \rightarrow \hat{f}(t, u)$ uniformly on $\mathbb{R} \times \mathcal{K}$ for any compact $\mathcal{K} \subset \mathbb{R}^{n}$. The topology on $\mathcal{H}(f)$ is the topology of uniform convergence on the sets $\mathbb{R} \times \mathcal{K}$ where $\mathcal{K} \subset \mathbb{R}^{n}$ is compact. It is obvious that the limiting equations satisfy an analog of (A1), (A3) and (A4). However, to make further developments we have to postulate that an analog of (A2) holds as in the following assumption.

(A7) For any $\hat{f} \in \mathcal{H}(f)$ each maximal solution of the equation

$$
\dot{u}=\hat{f}(t, u)
$$

with initial conditions $\left(t_{0}, u_{0}\right)$ exists at least on $\left[t_{0},+\infty\right)$.

Before giving proofs of the existence theorems we state some observations concerning the required properties of the projected equation and corresponding to it limiting ones. By (A1), (A7), (A3), (A4) we can apply the Smith reduction theorem to $(2 . \hat{f})$ to get the auxiliary function $\hat{\Phi}$ and the corresponding projected equation

$$
\dot{\zeta}=\Pi \hat{f}(t, \hat{\Phi}(t, \zeta))
$$

Now put $g(t, \zeta)=\Pi f\left(t, \Phi^{(0)}(t, \zeta)\right)$ and for any $\hat{g} \in \mathcal{H}(g)$ consider the equation

$$
\dot{\zeta}=\hat{g}(t, \zeta)
$$

It turns out that every limiting equation of the projected equation, i.e., Eq. (2. $\hat{g})$ is a projected equation for some limiting equation $(2 . \hat{f})$. This is the content of the following lemma. 
Lemma 2. Every Eq. (2. $\hat{g})$ has the form as in (2.16). In other words, for any $\hat{g} \in \mathcal{H}(g)$ there are $\hat{f} \in \mathcal{H}(f)$ and the corresponding $\hat{\Phi}$ such that $\hat{g}(t, \zeta)=\Pi \hat{f}(t, \hat{\Phi}(t, \zeta))$.

Proof. Suppose $\hat{f}_{k} \in \mathcal{H}(f)$ converges to some $\hat{f} \in \mathcal{H}(f)$ as $k \rightarrow \infty$. Repeating the argument as in the proof of Theorem 1 (condition (A5*) is necessary), we may show the convergence for corresponding auxiliary functions, namely, that $\hat{\Phi}_{k} \rightarrow \hat{\Phi}$. Now the required statement follows from this observation. Indeed, suppose $g\left(t+t_{k}, \zeta\right) \rightarrow \hat{g}(t, \zeta)$. By the Bochner theorem (see, for example, [9]) we may assume (taking a subsequence if necessary) that $f\left(t+t_{k}, u\right) \rightarrow \hat{f}(t, u)$ and $\Phi\left(t+t_{k}, \zeta\right) \rightarrow \hat{\Phi}(t, \zeta)$. It is evident that $\hat{g}(t, \zeta)=\Pi \hat{f}(t, \hat{\Phi}(t, \zeta)$ from the expression of $g$. But we have just proved that $\hat{\Phi}$ corresponds to Eq. $(2 . \hat{f})$. Therefore, $\hat{g}(t, \zeta)$ is the right-hand side of the projectied equation for the limiting equation $(2 . \hat{f})$.

From Lemma 2 it follows that any solution to Eqs. $(2 . \hat{g})$ exists on the whole real line, is unique and depends continously on the initial conditions due to the presence of this property for projected equations obtained by the Smith reduction theorem. However, to study almost periodic solutions we need the continuous dependence in the extended space, which we will postulate in the next assumption.

(A8) For all $t \geqslant 0$ the map $\left(\hat{g}, \zeta_{0}\right) \mapsto \zeta\left(t, \hat{g}, \zeta_{0}\right)$, where $\zeta\left(t, \hat{g}, \zeta_{0}\right)$ is a solution of $(2 . \hat{g})$ such that $\zeta\left(0, \hat{g}, \zeta_{0}\right)=\zeta_{0}$, is continuous as a map from $\mathcal{H}(g) \times \mathbb{R}^{j}$ to $\mathbb{R}^{j}$.

Also, we need the so-called positive uniform Lyapunov stability on compact sets as stated in the next assumption.

(A9) For every compact set $\mathcal{K} \subset \mathbb{R}^{n}$ and every $\varepsilon>0$ there is $\delta>0$ such that $\mid u\left(t, t_{0}, u_{0}\right)$ $-v\left(t, t_{0}, v_{0}\right) \mid<\varepsilon$ for all $t \geqslant t_{0}$ provided that $\left|u_{0}-v_{0}\right|<\delta$ and $u_{0}, v_{0} \in \mathcal{K}$.

Note that the projected equation inherits the positive uniform Lyapunov stability on compact sets since due to $(2.3)$ the auxiliary function $\Phi^{(0)}(t, \zeta)$ is globally Lipschitz in $\zeta$ uniformly in $t \in \mathbb{R}$.

Theorem 3. Let assumptions (A1)-(A4), (A5*) and (A7)-(A9) hold. Suppose that $j=2$ and (1.1) has a solution bounded on $\left[t_{0},+\infty\right)$; then (1.1) has an almost periodic solution $u^{*}(\cdot)$ with $\bmod _{\mathbb{Q}}\left(u^{*}\right) \subset \bmod _{\mathbb{Q}}(f)$. Moreover, any bounded solution is almost periodic.

Proof. Let $v(t)=v\left(t, t_{0}, v_{0}\right)$ be a solution to (1.1) bounded on $\left[t_{0},+\infty\right]$. We will show that there exists a solution bounded on $(-\infty,+\infty)$. For some $M>0$ we have $|v(t)| \leqslant M$ for all $t \geqslant t_{0}$. Let $\tau_{k}, k=1,2 \ldots$, be a sequence of $\varepsilon_{k}$-almost periods for $f(\cdot, u)$ uniformly on $|u| \leqslant M$ and such that $\tau_{k} \rightarrow+\infty, \varepsilon_{k} \rightarrow 0$ as $k \rightarrow \infty$. Consider $v_{k}(t):=v\left(t+\tau_{k}\right)$ which is a solution to $\left(2 \cdot \tau_{k}\right)$. Note that $v_{k}(t)$ is defined for $t \in\left(t_{0}-\tau_{k},+\infty\right)$ and $\left|v_{k}(t)\right| \leqslant M$. Therefore, the sequence $v_{k}(\cdot)$ is uniformly bounded and equicontinuous. By the Arzelà - Ascoli theorem and Cantor's diagonal argument we may subtract a subsequence which converges on every compact interval to some $v^{*}(\cdot)$. From the integral equation equivalent to (1.1) it is clear that $v^{*}(\cdot)$ is a solution to $(1.1)$ bounded on the whole real line. In particular, $v^{*}(\cdot)$ is amenable and, consequently, (A6) holds. The corresponding projected equation (1.3) (which is almost periodic due to Theorem 1) has a bounded solution $\Pi v^{*}(\cdot)$. From Theorem 5, p. 118 in [9] we get the existence of an almost periodic solution $\zeta^{*}(\cdot)$ with $\bmod _{\mathbb{Q}}\left(\zeta^{*}\right) \subset \bmod _{\mathbb{Q}}(g)$, where $g(t, \zeta)=\Pi f\left(t, \Phi^{(0)}(t, \zeta)\right)$ is the right-hand side of (1.3). Denote the corresponding almost periodic solution to $(1.1)$ by $u^{*}(\cdot)$, i. e. $u^{*}(t)=\Phi^{(0)}\left(t, \zeta^{*}(t)\right)$. From the proof of Theorem 2 and Theorem 1 we have $\bmod _{\mathbb{Q}}\left(\zeta^{*}\right) \subset \bmod _{\mathbb{Q}}(g) \Subset \bmod _{\mathbb{Q}}(f)$ and $\bmod _{\mathbb{Q}}\left(\Phi^{(0)}\right) \Subset \bmod _{\mathbb{Q}}(f)$. Therefore, $\bmod _{\mathbb{Q}}\left(u^{*}\right) \subset \bmod _{\mathbb{Q}}\left(\Phi^{(0)}, \zeta^{*}\right) \subset \bmod _{\mathbb{Q}}(\stackrel{\circ}{ })$. 
Moreover, by Theorem 5, p. 118 in [9] every bounded solution to the projected equation is almost periodic. By Theorem $1 \Phi^{(0)}$ takes these solutions to almost periodic solutions of (1.1). Therefore, any bounded solution to (1.1) is almost periodic.

REMARK 3. By $\left(\mathbf{A} 5^{*}\right)$ any almost periodic solution $u(\cdot)$ to $(1.1) \operatorname{must}$ have $\bmod _{\mathbb{Z}}(u) \supset \bmod _{\mathbb{Z}}(f)$ (however, as we stated in Remark 1, this inclusion may fail for more general right-hand sides) and by Theorem 2 for $j=2$ the dimension of the space generated by additional exponents is $\leqslant 1$.

To apply the existence theorem in dimension 3, we need an additional hypothesis.

(A10) There are a nonempty compact convex subset $\mathcal{B}_{0} \subset \mathbb{R}^{n}$ and a constant $T>0$ such that $u\left(t, 0, u_{0}\right) \in \mathcal{B}$ for all $t \geqslant T$ provided that $u_{0} \in \mathcal{B}_{0}$.

Theorem 4. Let assumptions (A1)-(A4), (A5*) and (A7)-(A10) hold. Suppose that $j=3$; then $(1.1)$ has an almost periodic solution $u^{*}(\cdot)$ with $\bmod _{\mathbb{Q}}\left(u^{*}\right) \subset \bmod _{\mathbb{Q}}(f)$.

Proof. From (A10) we have a solution bounded in $[0,+\infty)$, and as in the proof of Theorem 3, we can establish the existence of a solution to (1.1) that is bounded on the entire line (and therefore amenable). Thus, (1.1) satisfies (A6) and we can consider (1.3). It is clear that (1.3) satisfies (A9) and (A10) with $\Pi \mathcal{B}_{0}$ instead of $\mathcal{B}_{0}$. Therefore, Theorem 6, p. 118 in [9], gives us the existence of an almost periodic solution $\zeta^{*}(\cdot)$ with $\bmod _{\mathbb{Q}}\left(\zeta^{*}\right) \subset \bmod _{\mathbb{Q}}(g)$. By Theorem 1 the function $u^{*}(t):=\Phi^{(0)}\left(t, \zeta^{*}(t)\right)$ is an almost periodic solution to (1.1). As in the proof of Theorem 3 we have $\bmod _{\mathbb{Q}}\left(u^{*}\right) \subset \bmod _{\mathbb{Q}}(\stackrel{\circ}{f})$.

\section{Applications}

\subsection{Verification of main conditions}

Consider the following class of control systems:

$$
\dot{u}=A u+b \varphi\left(c^{*} u\right)+\stackrel{\circ}{f}(t)=f(t, u)
$$

with a real $n \times n$ matrix A; $n$-vectors $b$ and $c$; a scalar continuous function $\varphi(\cdot)$ and an $\mathbb{R}^{n}$-almost periodic function $f(t)$. Suppose that for some constants $0 \leqslant \varkappa_{1} \leqslant \varkappa_{2}<\infty$ the function $\varphi(\cdot)$ satisfies

$$
\varkappa_{1} \leqslant \frac{\varphi\left(v_{1}\right)-\varphi\left(v_{2}\right)}{v_{1}-v_{2}} \leqslant \varkappa_{2} \text { for all } v_{1}, v_{2} \in \mathbb{R} .
$$

Theorem 5. Let $\varphi$ from (3.1) satisfy (3.2); then assumptions (A1), (A2) and (A7) hold for (3.1). Moreover, if (A3) and (A4) are satisfied, then (A8) holds too.

Proof. It is obvious that (A1) holds. Since the nonlinearity $\varphi$ is globally Lipschitz, any solution of (3.1) can be extended to the whole real line. Therefore, (A2) is satisfied.

Now let $\hat{f} \in \mathcal{H}(f)$. It is clear that $\hat{f}(t, u)=A u+b \varphi\left(c^{*} u\right)+\hat{\hat{f}}(t)$, where $\hat{\dot{f}} \in \mathcal{H}(\dot{f})$. By the previous arguments we have (A7).

Suppose (A3) and (A4) are satisfied for (3.1) and, consequently, for any system $(2 . \hat{f})$, i.e., we can apply the Smith reduction theorem and consider the projected equations $(2 . \hat{g})$. In our case $g(t, \zeta)=\Pi h\left(\Phi^{(0)}(t, \zeta)\right)+\Pi \AA(t)$, where $h(u)=A u+b \varphi\left(c^{*} u\right)$ is globally Lipschitz. Now let $t \geqslant 0$ be fixed and suppose that $\hat{g}_{k} \in \mathcal{H}(g)$ converges to some $\hat{g} \in \mathcal{H}(g), \zeta_{0}^{(k)}$ converges to $\zeta_{0} \in \mathbb{R}^{j}$. Let us show that $\zeta_{k}(t):=\zeta\left(t, \hat{g}_{k}, \zeta_{0}^{(k)}\right)$ converges to $\zeta(t):=\zeta\left(t, \hat{g}, \zeta_{0}\right)$. Indeed, 
consider $\Delta_{k}(s):=\zeta_{k}(s)-\zeta(s)$. We have the equality

$$
\Delta(t)=\Delta(0)+\int_{0}^{t}\left(\hat{g}_{k}\left(s, \zeta_{k}(s)\right)-\hat{g}(s, \zeta(s))\right) d s .
$$

In the identity

$$
\hat{g}_{k}\left(s, \zeta_{k}(s)\right)-\hat{g}(s, \zeta(s))=\left[\hat{g}_{k}\left(s, \zeta_{k}(s)\right)-\hat{g}_{k}(s, \zeta(s))\right]+\left[\hat{g}_{k}(s, \zeta(s))-\hat{g}(s, \zeta(s))\right]
$$

the second term in square brackets is small due to the convergence of $\hat{g}_{k}$. To deal with the first term we use Lemma 2 to get the functions $\hat{\hat{f}}_{n} \in \mathcal{H}(\stackrel{\circ}{f})$ and $\hat{\Phi}_{k}$ such that $\hat{g}_{k}(s, \zeta)=\Pi h\left(\hat{\Phi}_{k}(s, \zeta)\right)+$ $+\Pi \hat{\hat{f}}_{k}(s)$. Since $\Pi, h$ and $\hat{\Phi}_{k}$ are globally Lipschitz, ${ }^{6}$ there exists a constant $C>0$ (independent of $k$ ) such that

$$
\left|\hat{g}_{k}\left(s, \zeta_{k}(s)\right)-\hat{g}_{k}(s, \zeta(s))\right| \leqslant C\left|\zeta_{k}(s)-\zeta(s)\right|=C|\Delta(s)| .
$$

Therefore, for some sequence $\varepsilon_{k} \rightarrow 0$ we have

$$
\left|\Delta_{k}(t)\right| \leqslant \varepsilon_{k}+C \int_{0}^{t}\left|\Delta_{k}(s)\right| d s .
$$

From the Gronwall inequality we conclude that $\Delta_{k}(t) \rightarrow 0$ as $k \rightarrow \infty$. The proof is finished.

Now let $W(p):=c^{*}(A-p I)^{-1} b$ be the transfer function of the linear part of (3.1). Suppose that for some $\nu>0$ the matrix $A+\nu I$ has $j$ eigenvalues with positive real parts and $n-j$ eigenvalues with negative real parts and the pair $(A+\nu I, b)$ is stabilizable ${ }^{7}$. In this case the following frequency domain condition can be checked to get the matrix $P$ such that (3.1) will satisfy assumptions (A3) and (A4).

(F1) $\operatorname{Re}\left(\left[1+\varkappa_{1} W(i \omega-\nu)\right]^{*}\left[1+\varkappa_{2} W(i \omega-\nu)\right]\right)>0$ for $\omega \in[-\infty, \infty]$.

This condition is well-known in control theory $([3,8,16])$. For the proof of this fact we refer to [16]. It is worth noting that the above properties can be verified for control systems with several nonlinearities (see [16]).

\subsection{Sensitive dependence on initial conditions}

We start with the following

Theorem 6. Suppose that (F1) is satisfied for (3.1) with the matrix $A+\nu I$ having $j=2$ eigenvalues with positive real parts. If (A9) is satisfied, then any bounded solution to any limiting equation for (3.1) is almost periodic.

Proof. We apply Theorem 3 to each of the limiting equations for (3.1). This is possible since (A9) automatically holds for limiting equations.

Now we consider Chua's oscillator with an almost periodic force:

$$
\left\{\begin{array}{l}
\dot{x}=\alpha(y-x-h(x)) \\
\dot{y}=x-y+z \\
\dot{z}=-(\beta y+\gamma z)+f(t)
\end{array}\right.
$$

\footnotetext{
${ }^{6}$ For $\hat{\Phi}_{k}$ by (2.3) the Lipschitz constant depends only on the matrix $P$ in (A3).

${ }^{7} \mathrm{~A}$ pair of matrices $(A, B)$ with sizes $n \times n$ and $n \times k$ is called stabilizable if there exists an $n \times k$ matrix $K$ such that any eigenvalue of the matrix $A+B K^{*}$ is located to the left of the imaginary axis.
} 
Here $h(x)=m_{1} x+\frac{1}{2}\left(m_{0}-m_{1}\right)(|x+1|-|x-1|)$ and $\alpha, \beta, \gamma, m_{0}, m_{1}$ are parameters. System (3.7) can be considered as $(3.1)$, where $b=[\alpha, 0,0]^{T}, c=[1,0,0]^{T},-\varphi(\sigma)=m_{1} \sigma+\frac{1}{2}\left(m_{0}-m_{1}\right) \times$ $(|\sigma+1|-|\sigma-1|)$ and

$$
A=\left[\begin{array}{ccc}
-\alpha & \alpha & 0 \\
1 & -1 & 1 \\
0 & -\beta & -\gamma
\end{array}\right]
$$

In [15] the so-called strange nonchaotic attractor in (3.7) was discovered for the values of parameters $^{8}: \alpha=5.8333, \beta=12.8012, \gamma=0, m_{0}=-1.1862$ and $m_{1}=-0.64$. We will show that with these parameters system (3.7) satisfies (F1) with $j=2$. In this case the matrix $A$ has the eigenvalues: $\lambda_{1} \approx-6.607, \lambda_{2,3} \approx-0.113 \pm 3.360$. We take $\nu=5$ and $\varkappa_{1}=-m_{1}=$ $=0.64, \varkappa_{2}=-m_{0}=1.1862$ in (3.2). It can be checked that for these parameters we have $\operatorname{Re}\left(\left[1+\varkappa_{1} W(i \omega-\nu)\right]^{*}\left[1+\varkappa_{2} W(i \omega-\nu)\right]\right)>0, \omega \in \mathbb{R}$. Therefore, condition (F1) holds. The stabilizability of $(A+\nu I, b)$ in this case can be verified too, for example, by checking its controllabity $^{9}$ (this fact is well-known in control theory, [16]).

We say that system (3.1) has sensitive dependence on initial conditions if the contrary to (A9) holds, i.e., the system is not uniformly positive Lyapunov stable. It was proved in [5] that certain classes of classical strange nonchaotic attractors (see [6]) have sensitive dependence on initial conditions (in a much stronger sense than considered in our work). To understand this phenomenon, for example, in model (3.7), one may need to know some structure of the attractor in the absence of sensitivity. Thus, if one supposes that the system has no sensitive dependence on initial conditions (in the sense given above), then from Theorem 6 we have the property that all bounded solutions are almost periodic. In particular, any trajectory on the attractor is almost periodic. This fact together with some knowledge of the structure of these almost periodic solutions may contradict to other established properties of the attractor.

Moreover, under the conditions of Theorem 6 something more can be shown. Namely, not only all bounded solutions are almost periodic, but every solution which remains bounded in the future converges to one of these almost periodic solutions as $t \rightarrow+\infty$. Thus, under the condition of the uniformly positive Lyapunov stability the only observable regimes of the system are almost periodic. We will deal with this problem elsewhere in a more expanded form.

\section{References}

[1] Anikushin, M. M., On the Liouville Phenomenon in Estimates of Fractal Dimensions of Forced Quasi-Periodic Oscillations, Vestnik St. Petersb. Univ. Math., 2019, vol.52, no. 3 (in press).

[2] Anikushin, M. M., Dimensional Aspects of Almost Periodic Dynamics, in [8] (2019).

[3] Burkin, I. M., Method of "Transition into Space of Derivatives": 40 Years of Evolution, Differ. Equ., 2015, vol.51, no.13, pp. 1717-1751; see also: Differ. Uravn., 2015, no. 3, pp. 51-93.

[4] Cartwright, M. L., Almost Periodic Differential Equations and Almost Periodic Flows, J. Differential Equations, 1969, vol. 5, pp. 167-181.

${ }^{8}$ The almost periodic perturbation is considered to be 2-frequency quasi-periodic of type $f(t)=$ $=a \sin \left(\omega_{1} t\right)+b \sin \left(\omega_{2} t\right)$.

${ }^{9}$ The pair $(A, b)$, where $A$ is an $n \times n$-matrix and $b$ is an $n$-vector, is called controllable if the matrix $\left[b, A b, \ldots, A^{n-1} b\right]$ has maximal rank. 
[5] Glendinning, P., Jäger, T.H., and Keller, G., How Chaotic Are Strange Non-Chaotic Attractors?, Nonlinearity, 2006, vol. 19, no. 9, pp. 2005-2022.

[6] Feudel, U., Kuznetsov, S., and Pikovsky, A., Strange Nonchaotic Attractors: Dynamics between Order and Chaos in Quasiperiodically Forced Systems, World Sci. Ser. Nonlinear Sci. Ser. A Monogr. Treatises, vol. 56, Hackensack, N.J.: World Sci., 2006.

[7] Fink, A. M., Almost Periodic Differential Equations, Lecture Notes in Math., vol.377, Berlin: Springer, 1974.

[8] Leonov, G. A., Kuznetsov, N. V., and Reitmann, V., Attractor Dimension Estimates for Dynamical Systems: Theory and Computation, Cham: Springer, 2019 (in press).

[9] Levitan, B. M. and Zhikov, V. V., Almost Periodic Functions and Differential Equations, Cambridge: Cambridge Univ. Press, 1982.

[10] O'Brien, G. C., The Frequencies of Almost Periodic Solutions of Almost Periodic Differential Equations, J. Austral. Math. Soc., 1974, vol. 17, pp. 332-344.

[11] Popov, S. and Reitmann, V., Frequency Domain Conditions for Finite-Dimensional Projectors and Determining Observations for the Set of Amenable Solutions, Discrete Contin. Dyn. Syst., 2014, vol. 34, no. 1, pp. 249-267.

[12] Robinson, J. C., Inertial Manifolds and the Strong Squeezing Property, in Nonlinear Evolution Equations 8 Dynamical Systems: NEEDS'94 (Los Alamos, N.M.), River Edge, N.J.: World Sci., 1995, pp. $178-187$.

[13] Smith, R.A., Massera's Convergence Theorem for Periodic Nonlinear Differential Equations, J. Math. Anal. Appl., 1986, vol.120, no.2, pp.679-708.

[14] Smith, R. A., Convergence Theorems for Periodic Retarded Functional-Differential Equations, Proc. London Math. Soc. (3), 1990, vol.60, no. 3, pp. 581-608.

[15] Suresh, K., Prasad, A., and Thamilmaran, K., Birth of Strange Nonchaotic Attractors through Formation and Merging of Bubbles in a Quasiperiodically Forced Chua's Oscillator, Phys. Lett. A, 2013, vol. 377, no. 8, pp. 612-621.

[16] Yakubovich, V.A., Leonov, G. A., and Gelig, A. Kh., Stability of Stationary Sets in Control Systems with Discontinuous Nonlinearities, Ser. Stab. Vib. Control Syst. Ser. A, vol. 14, River Edge, N.J.: World Sci., 2004. 